\title{
SELF-CONSISTENT MASSIVE DISKS IN TRIAXIAL DARK MATTER HALOS
}

\author{
Jeremy Bailin, ${ }^{1,2}$ Joshua D. Simon, ${ }^{3}$ Alberto D. Bolatto, ${ }^{4}$ Brad K. Gibson, ${ }^{5}$ and Chris Power ${ }^{1}$ \\ Received 2007 April 2; accepted 2007 June 9
}

\begin{abstract}
Galactic disks in triaxial dark matter halos become deformed by the elliptical potential in the plane of the disk in such a way as to counteract the halo ellipticity. We develop a technique to calculate the equilibrium configuration of such a disk in the combined disk-halo potential, which is based on the method of Jog but accounts for the radial variation in both the halo potential and the disk ellipticity. This crucial ingredient results in qualitatively different behavior of the disk: the disk circularizes the potential at small radii, even for a reasonably low disk mass. This effect has important implications for proposals to reconcile cuspy halo density profiles with low surface brightness galaxy rotation curves using halo triaxiality. The disk ellipticities in our models are consistent with observational estimates based on two-dimensional velocity fields and isophotal axis ratios.
\end{abstract}

Subject headings: dark matter — galaxies: halos — galaxies: kinematics and dynamics — galaxies: spiral — galaxies: structure - methods: numerical

Online material: color figures

\section{INTRODUCTION}

Galaxies are thought to be surrounded by large dark matter halos. These halos are much more massive than the visible components of galaxies and dominate much of the dynamics. Although dark matter halos are often assumed to be spherical for simplicity, the halos that form in cosmological simulations are quite flattened, with typical intermediate axis ratios of $b / a \sim 0.8$ and minor axis ratios of $c / a \sim 0.6$, with some systematic variation depending on the mass of the halo and the radius at which the shape is measured (Warren et al. 1992; Jing \& Suto 2002; Bailin \& Steinmetz 2005; Allgood et al. 2006). This nonsphericity is a testable prediction of cosmological models.

Simulations of disk galaxy formation within dark matter halos find that the presence of the disk modifies the shape of the halo, reducing the halo triaxiality (Dubinski 1994; Kazantzidis et al. 2004; Bailin et al. 2005; Berentzen \& Shlosman 2006). However, as long as the final shape of the halo retains some ellipticity in the plane of the disk, the dynamics and shape of the disk will be affected by the deviations from axisymmetry (e.g., Gerhard \& Vietri 1986; Schoenmakers et al. 1997).

Observations indicate that many disks do indeed have small but nonzero ellipticities. Evidence for elliptical disks comes from harmonic decomposition of galaxy photometry (Rix \& Zaritsky 1995), harmonic decomposition of two-dimensional velocity fields (Schoenmakers et al. 1997; Simon et al. 2005), and statistical analysis of the distribution of projected shapes (Ryden 2006). These results qualitatively confirm that galactic dark matter halos are elliptical; precision measurements could provide direct constraints on the shapes of the halos.

Recently, Hayashi \& Navarro (2006, hereafter HN06) proposed that elliptical orbits within the disk produced by the triaxiality of

\footnotetext{
1 Centre for Astrophysics and Supercomputing, Swinburne University of Technology, Mail H39, P.O. Box 218, Hawthorn, Victoria, 3122, Australia.

2 Current address: Department of Physics and Astronomy, ABB-241, McMaster University, 1280 Main Street West, Hamilton, ON L8S 4M1, Canada.

3 Department of Astronomy, California Institute of Technology, 1200 East California Boulevard, MS 105-24, Pasadena, CA 91125.

4 Department of Astronomy and Radio Astronomy Laboratory, University of California, Berkeley, CA 94720.

${ }^{5}$ Centre for Astrophysics, University of Central Lancashire, Preston, PR1 2HE, UK.
}

the halo could reconcile cuspy density profiles that form in cosmological simulations (Navarro et al. 1996, hereafter NFW96) with observed rotation curves of low surface brightness (LSB) galaxies, which often appear to require halos with constant density cores (e.g., de Blok et al. 2001). This analysis did not take into account the self-gravity of the disk. In galaxies with massive disks, the gravity of the disk contributes to the net potential, and the dynamics of the disk are determined by a combination of the halo and the disk itself. In order to draw conclusions about the shape of the halo from the measured dynamics of the disk, we must determine self-consistently both how the disk is perturbed by the potential and how the perturbed disk contributes to the potential.

An elegant method to carry out these calculations was proposed by Jog (2000, hereafter J2K; see also Jog 1997, 1999). By assuming a logarithmic halo potential with a small constant elliptical perturbation and an exponential disk with a small constant elliptical response, J2K solved for the self-consistent response. She demonstrated that the disk response dilutes the ellipticity of the potential most strongly at 1.42 disk scale lengths.

There are a number of simplifying assumptions in $\mathrm{J} 2 \mathrm{~K}$ that require examination. The most important assumption is that both the halo perturbation and the disk response are constant with radius. In contrast, HN06 demonstrated that a radially varying perturbation is required to reconcile LSB long-slit rotation curves with cuspy halo profiles. Indeed, cosmological simulations predict a radially varying perturbation in the halo potential; even if halos had isodensity surfaces of constant ellipticity, the shape of the potential would vary with radius, and Hayashi et al. (2007, hereafter HNS07), who directly measured the shapes of isopotential surfaces of cosmological halos, found even stronger variation with radius. The response of the disk is also not expected to be uniform in a radially varying potential.

In this paper we generalize the method of $\mathrm{J} 2 \mathrm{~K}$ to the more realistic case of radially varying halo perturbations and radially varying disk responses. In $\S 2$ we detail the method for determining the disk shape and dynamics. In $\S 3$ we use this method to determine the shapes and dynamics of disks in sample triaxial halos and demonstrate how the results depend on the properties of the disks and halos. Section 4 discusses our results in the context of observations that directly probe disk ellipticity, and in $\S 5$ we present our conclusions. 


\section{METHOD}

\subsection{Outline}

Our method, which is based closely on $\mathrm{J} 2 \mathrm{~K}$, is as follows.

1. Calculate the axisymmetric component of the potential and the elliptical perturbation in the potential induced by the triaxial halo $(\S 2.2)$.

2. Calculate the closed orbits and corresponding disk ellipticity for a given net perturbation to the potential ( $\$ \S 2.3$ and 2.4).

3 . Calculate the elliptical perturbation in the potential induced by a given disk ellipticity $(\S 2.5)$.

4. Solve for the form of the net potential perturbation that satisfies all of the above constraints ( $\S 2.6)$.

Throughout this procedure, the halo is kept fixed, i.e., it does not respond to the presence of the disk. Therefore, the potential that should be used in the calculation is the real shape of the halo in which the disk lies, which is less triaxial than the shape the halo would have in the absence of baryonic processes (Kazantzidis et al. 2004; Bailin et al. 2005; Berentzen \& Shlosman 2006).

The main differences between this work and $\mathrm{J} 2 \mathrm{~K}$ are:

1. Where $\mathrm{J} 2 \mathrm{~K}$ assumes a logarithmic potential for the halo, we evaluate the radial form of the potential directly from a density distribution motivated by cosmological simulations.

2 . Where $\mathrm{J} 2 \mathrm{~K}$ assumes a constant perturbation to the halo potential, we allow the perturbation to vary with radius and either evaluate it directly from a triaxial density distribution motivated by cosmological simulations or use parameterizations developed from measurements of halos in cosmological simulations.

3. Where $\mathrm{J} 2 \mathrm{~K}$ assumes that the disk responds with a constant ellipticity, we allow the disk ellipticity to vary with radius.

Whenever we carry out numerical calculations in this paper, all radially varying functions are tabulated on a radial grid sampled at 100 radii $R_{i}$ spaced logarithmically between 0.1 and $100 \mathrm{kpc}$ in order to finely sample the inner region of the disk where the quantities vary most rapidly; 50 grid points lie at $R<1 \mathrm{kpc}$. The functions are linearly interpolated between grid points when their values are required at arbitrary radii.

\subsection{Axisymmetric Potential and Halo Perturbation}

For the perturbative approach, we assume that within the plane of the disk (which we take to be $z=0$ for simplicity), the total potential can be written as

$$
\begin{aligned}
\Phi(R, \theta) & =\Phi_{0}(R)+\Phi_{\text {pert }}(R, \theta) \\
& =\Phi_{0}(R)\left[1+f_{\text {pert }}(R) \cos m \theta\right],
\end{aligned}
$$

where $R, \theta$, and $z$ are the cylindrical coordinates. For the purposes of this paper, we assume an elliptical perturbation (i.e., $m=2$ ) from now on (see the Appendix for a discussion of the $m=4$ mode). We assume that $f_{\text {pert }}(R)$ is small and varies slowly with $R$. Note that for $f_{\text {pert }}>0$, isopotentials are elongated along the $x$-axis, and closed orbits in the disk are elongated along the $y$-axis.

Both the disk and halo contribute to both the axisymmetric and $m=2$ components of the potential:

$$
\begin{aligned}
& \Phi_{0}(R)=\Phi_{0}^{\text {halo }}(R)+\Phi_{0}^{\text {disk }}(R), \\
& f_{\text {pert }}(R)=f_{\text {pert }}^{\text {halo }}(R)+f_{\text {pert }}^{\text {disk }}(R) .
\end{aligned}
$$

To first order, the $m=2$ perturbation in the potential induces an $m=2$ perturbation in the surface density distribution of the otherwise exponential disk (see the Appendix for a justification of our decision to neglect the higher order terms),

$$
\Sigma(R, \theta)=\Sigma_{0} \exp \left\{-\frac{R}{R_{d}}\left[1-\frac{\epsilon_{\mathrm{disk}}(R)}{2} \cos 2 \theta\right]\right\} .
$$

We assume $\epsilon_{\text {disk }}(R)$, the ellipticity of the isodensity ellipse, is small and varies slowly with $R$. The axisymmetric component of the disk potential is given by

$$
\Phi_{0}^{\text {disk }}(R)=-\pi G \Sigma_{0} R\left[I_{0}(y) K_{1}(y)-I_{1}(y) K_{0}(y)\right],
$$

where $I$ and $K$ are modified Bessel functions ( $y=R / 2 R_{d}$; see Freeman 1970; Binney \& Tremaine 1987, eq. [2-168]).

Given the density distribution of the dark matter halo, both axisymmetric and $m=2$ components of the halo potential can be evaluated. If the isodensity surfaces of the halo are self-similar ellipsoids, then the halo density can be written as

$$
\rho(x, y, z)=\rho(s)
$$

where

$$
s^{2}=\left(\frac{x}{a}\right)^{2}+\left(\frac{y}{b}\right)^{2}+\left(\frac{z}{c}\right)^{2}
$$

For example, we can use an NFW96 form for the density (NFW96; Jing \& Suto 2002),

$$
\rho(s)=\frac{\rho_{0}}{\left(s / r_{s}\right)\left[1+\left(s / r_{s}\right)\right]^{2}} .
$$

We make no assumptions about the relative magnitudes of $a, b$, and $c$; they are simply the relative axis ratios along the $x$-, $y$-, and $z$-axes, respectively. Therefore, the disk, which lies in the $x-y$ plane, can be oriented in any of the principal planes of the halo.

We calculate the halo potential along the $x$-axis, $\Phi_{x}^{\text {halo }}(R) \equiv$ $\Phi^{\text {halo }}(R, 0,0)$, and along the $y$-axis, $\Phi_{y}^{\text {halo }}(R) \equiv \Phi^{\text {halo }}(0, R, 0)$, by numerically integrating equation (2-99) of Binney \& Tremaine (1987) using $\rho(s)$ given in equation (8). This allows us to calculate $\Phi_{0}^{\text {halo }}$ and $f_{\text {pert }}^{\text {halo }}$ as

$$
\begin{gathered}
\Phi_{0}^{\text {halo }}(R)=\frac{1}{2}\left[\Phi_{x}(R)+\Phi_{y}(R)\right], \\
f_{\text {pert }}^{\text {halo }}(R)=\frac{1}{2 \Phi_{0}(R)}\left[\Phi_{x}(R)-\Phi_{y}(R)\right] .
\end{gathered}
$$

\subsection{Closed Orbits}

In a triaxial potential, dissipative gas settles on stable closed loop orbits when such orbits exist (El-Zant 2001). This is the case throughout the potential of a centrally concentrated mass profile such as the NFW96 profile. Therefore, the structure of a galactic disk, which consists of gas clouds and stars formed within those gas clouds, is determined by the form of the closed orbits. These have been examined in detail by Schoenmakers et al. (1997) and simplified into a convenient form by HN06. These previous derivations have assumed that the perturbation to the potential is constant over the radial excursion of an orbit, which is not the case for the radially varying perturbations that we wish to study. We have therefore rederived the equations for closed orbits within a radially 
varying perturbation from the equations of motion. The orbits follow

$$
\begin{aligned}
& R=R_{0}\left(1-\frac{f_{\text {pert }} a_{12}}{2} \cos 2 \theta_{0}\right), \\
& \theta=\theta_{0}+\frac{a_{12}+a_{32}}{2 m} f_{\text {pert }} \sin 2 \theta_{0},
\end{aligned}
$$

with velocities

$$
\begin{gathered}
V_{R}=V_{c} f_{\text {pert }} a_{12} \sin 2 \theta_{0}, \\
V_{\theta}=V_{c}\left(1+\frac{f_{\text {pert }} a_{32}}{2} \cos 2 \theta_{0}\right),
\end{gathered}
$$

where $R_{0}$ and $\theta_{0}$ define the guiding center of the orbit, $\theta_{0}=\Omega_{0} t$, and the following functions of $\Phi_{0}(R)$ are evaluated at $R_{0}$ :

$$
\begin{gathered}
\Omega_{0}(R)=\sqrt{\frac{1}{R} \frac{d \Phi_{0}}{d R}}, \\
V_{c}(R)=R \Omega_{0}(R), \\
V_{\mathrm{esc}}(R)=\sqrt{2\left|\Phi_{0}\right|}, \\
g_{m}(R)=\frac{1}{\Omega_{0}^{2}} \frac{d^{2} \Phi_{0}}{d R^{2}}-\left(m^{2}-3\right), \\
a_{1 m}(R)=\frac{2}{g_{m}(R)}\left[1-\frac{V_{\mathrm{esc}}^{2}}{V_{c}^{2}}\left(1+\frac{1}{2} \frac{R}{f_{\mathrm{pert}}} \frac{d f_{\mathrm{pert}}}{d R}\right)\right], \\
a_{3 m}(R)=a_{1 m}(R)+\frac{V_{\mathrm{esc}}^{2}}{V_{c}^{2}} .
\end{gathered}
$$

The coefficients $a_{1 m}$ and $a_{3 m}$ quantify the degree to which the radius $R$ and angular velocity $V_{\theta}$, respectively, which are constant for a circular orbit, vary for a unit perturbation to the potential. Our expressions differ from those in HNO6 for the following reasons. (1) We have taken the radial variation of the perturbation into account in our derivation of equation (19). (2) We have generalized the expression for $g_{m}(R)$ to be valid for all $m$, while the expression in HN06 is specific to $m=2$. (3) We have removed the factor of $f_{\text {pert }}$ from the definitions of $a_{1 m}$ and $a_{3 m}$ for convenience later (note that these quantities still depend implicitly on $f_{\text {pert }}$ through its derivative). (4) We have generalized the expression for $a_{3 m}$ to be valid for all potential profiles, while the expression in HN06 is only valid when $g_{2}(R)=-3$, which is not the case in the inner regions of an NFW96 potential.

Given the tabulated values of $\Phi_{0}$, these functions can be evaluated at the grid points $R_{i}$. Because $\Phi_{0}$ has been calculated from analytic functions, the tabulated values are relatively free of noise, and even the numerical second derivative does not contain large fluctuations. Note that the closed orbits are elongated perpendicular to the isopotential contours.

\subsection{Disk Ellipticity}

The disk must satisfy the continuity equation. In cylindrical coordinates,

$$
\frac{\partial}{\partial R}\left[R \Sigma(R, \theta) V_{R}(R, \theta)\right]+\frac{\partial}{\partial \theta}\left[\Sigma(R, \theta) V_{\theta}(R, \theta)\right]=0 .
$$

We substitute $\Sigma(R, \theta)$ from equation (4), $V_{R}(R, \theta)$ from equation (13), and $V_{\theta}(R, \theta)$ from equation (14). To first order in the small quantities $f_{\text {pert }}, \epsilon_{\text {disk}}$, and their derivatives,

$$
\begin{aligned}
\frac{R}{R_{d}} \epsilon_{\text {disk }}(R)= & f_{\text {pert }}(R) \\
& \times\left[a_{12}(R)\left(1-\frac{R}{R_{d}}+\frac{R}{V_{c}} \frac{d V_{c}}{d R}\right)-a_{32}(R)\right] .
\end{aligned}
$$

The neglected second-order terms induce small $m=4$ perturbations in the disk; see the Appendix for details. ${ }^{6}$ Equation (22) provides us with a relationship between the radial profile of the potential (embodied in $a_{12}, a_{32}$, and $V_{c}$ ), the strength of the perturbation in the net potential $\left(f_{\text {pert }}\right)$, and the ellipticity of the disk $\left(\epsilon_{\text {disk }}\right)$.

\subsection{Disk Perturbation Potential}

The ellipticity of the disk generates an $m=2$ perturbation to the disk potential. We calculate this as (Binney \& Tremaine 1987, eq. [2P-8])

$$
\begin{aligned}
& \Phi_{\text {pert }}^{\text {disk }} \equiv \Phi^{\text {disk }}-\Phi_{0}^{\text {disk }}=-G \sum_{m=-\infty}^{\infty} \exp (i m \theta) \\
& \times \int_{0}^{\infty} J_{m}(k R) \exp (-k|z|) d k \int_{0}^{\infty} J_{m}\left(k R^{\prime}\right) R^{\prime} d R^{\prime} \\
& \times \int_{0}^{2 \pi}\left[\Sigma\left(R^{\prime}, \theta^{\prime}\right)-\Sigma_{0} \exp \left(-R^{\prime} / R_{d}\right)\right] \exp \left(-i m \theta^{\prime}\right) d \theta^{\prime} .
\end{aligned}
$$

We restrict ourselves to the plane $z=0$, corresponding to an infinitely thin disk.

For small perturbations, the perturbed surface density is

$$
\Sigma\left(R^{\prime}, \theta^{\prime}\right)-\Sigma_{0} e^{-R^{\prime} / R_{d}} \approx \Sigma_{0} e^{-R^{\prime} / R_{d}} \frac{R^{\prime}}{R_{d}} \frac{\epsilon_{\mathrm{disk}}\left(R^{\prime}\right)}{2} \cos 2 \theta^{\prime} .
$$

Substituting equation (24) into equation (23), we note that the integral over $d \theta^{\prime}$ vanishes except when $m= \pm 2$. Since $\int_{0}^{2 \pi} \cos 2 \theta^{\prime} e^{ \pm i 2 \theta^{\prime}} d \theta^{\prime}=\pi, e^{i x}+e^{-i x}=2 \cos x$, and $J_{2}(x)=J_{-2}(x)$, we find

$$
\begin{aligned}
& \Phi_{\mathrm{pert}}^{\mathrm{disk}}(R, \theta)=-\pi G \Sigma_{0} \cos 2 \theta \int_{0}^{\infty} J_{2}(k R) d k \\
& \times \int_{0}^{\infty} J_{2}\left(k R^{\prime}\right) R^{\prime} \exp \left(-\frac{R^{\prime}}{R_{d}}\right) \frac{R^{\prime}}{R_{d}} \epsilon_{\mathrm{disk}}\left(R^{\prime}\right) d R^{\prime} .
\end{aligned}
$$

We can express $\left(R^{\prime} / R_{d}\right) \epsilon_{\text {disk }}\left(R^{\prime}\right)$ in the final integral in terms of $f_{\text {pert }}, a_{12}, a_{32}$, and $V_{c}$ using equation (22):

$$
\begin{aligned}
& \Phi_{\text {pert }}^{\text {disk }}(R, \theta)=-\pi G \Sigma_{0} \cos 2 \theta \int_{0}^{\infty} J_{2}(k R) d k \\
& \times \int_{0}^{\infty} J_{2}\left(k R^{\prime}\right) R^{\prime} \exp \left(-\frac{R^{\prime}}{R_{d}}\right) f_{\text {pert }}\left(R^{\prime}\right) \\
& \times\left\{a_{12}\left(R^{\prime}\right)\left[1-\frac{R^{\prime}}{R_{d}}+\frac{R^{\prime}}{V_{c}\left(R^{\prime}\right)} \frac{d V_{c}\left(R^{\prime}\right)}{d R^{\prime}}\right]-a_{32}\left(R^{\prime}\right)\right\} d R^{\prime} .
\end{aligned}
$$

Because we do not know a priori the net perturbation $f_{\text {pert }}(R)$, we cannot immediately evaluate these integrals. However, if we

\footnotetext{
${ }^{6}$ We have also omitted the term in eq. (22) that is proportional to $R d\left(f_{\text {pert }} a_{12}\right) / d R$; however, its effect is negligible.
} 
can find a function $f_{\text {pert }}^{\text {prox }}$ whose form is similar to $f_{\text {pert }}$, i.e., if $f_{\text {pert }} / f_{\text {pert }}^{\text {prox }}$ is a slowly varying function of $R$, then we can approximate the potential as

$$
\begin{aligned}
& \Phi_{\text {pert }}^{\text {disk }}(R, \theta) \approx-\frac{f_{\text {pert }}(R)}{f_{\text {pert }}^{\text {proxy }}(R)} \pi G \Sigma_{0} \cos 2 \theta \int_{0}^{\infty} J_{2}(k R) d k \\
& \times \int_{0}^{\infty} J_{2}\left(k R^{\prime}\right) R^{\prime} \exp \left(-\frac{R^{\prime}}{R_{d}}\right) f_{\text {pert }}^{\text {proxy }}\left(R^{\prime}\right) \\
& \times\left\{a_{12}\left(R^{\prime}\right)\left[1-\frac{R^{\prime}}{R_{d}}+\frac{R^{\prime}}{V_{c}\left(R^{\prime}\right)} \frac{d V_{c}\left(R^{\prime}\right)}{d R^{\prime}}\right]-a_{32}\left(R^{\prime}\right)\right\} d R^{\prime}
\end{aligned}
$$

Note that $a_{12}\left(R^{\prime}\right)$ and $a_{32}\left(R^{\prime}\right)$ depend implicitly on $f_{\text {pert }}^{\text {prox }}\left(R^{\prime}\right)$ through its derivative. A first approximation can be obtained by setting $f_{\text {pert }}^{\text {proxy }}=f_{\text {pert }}^{\text {halo }}$, whose values have been tabulated from equation (10). Because the integral over $R^{\prime}$ is independent of $R$ and the integral over $k$ is independent of $R^{\prime}$, the integrals can be evaluated independently on fine grids of $k$ and $R$, respectively. Using this technique, we calculate

$$
\begin{aligned}
& \eta(R) \equiv \frac{\pi G \Sigma_{0}}{f_{\text {pert }}^{\text {proxy }}(R)} \int_{0}^{\infty} J_{2}(k R) d k \\
& \times \int_{0}^{\infty} J_{2}\left(k R^{\prime}\right) R^{\prime} \exp \left(-\frac{R^{\prime}}{R_{d}}\right) f_{\mathrm{pert}}^{\text {proxy }}\left(R^{\prime}\right) \\
& \times\left\{a_{12}\left(R^{\prime}\right)\left[1-\frac{R^{\prime}}{R_{d}}+\frac{R^{\prime}}{V_{c}\left(R^{\prime}\right)} \frac{d V_{c}\left(R^{\prime}\right)}{d R^{\prime}}\right]-a_{32}\left(R^{\prime}\right)\right\} d R^{\prime}
\end{aligned}
$$

at each grid point $R_{i}$. The perturbation potential due to the disk is then given by

$$
\Phi_{\text {pert }}^{\text {disk }}(R, \theta)=-f_{\text {pert }}(R) \eta(R) \cos 2 \theta .
$$

Expressed in this form, the meaning of $\eta(R)$ becomes clear: it is the magnitude of the disk response to a unit perturbation in the potential.

\subsection{Self-Consistent Solution}

For clarity, we repeat here the important equations,

$$
\begin{aligned}
\Phi_{\text {pert }}(R, \theta) & =\Phi_{0}(R) f_{\text {pert }}(R) \cos 2 \theta \\
= & \Phi_{\text {pert }}^{\text {halo }}(R, \theta)+\Phi_{\text {pert }}^{\text {disk }}(R, \theta), \\
\Phi_{\text {pert }}^{\text {halo }}(R, \theta) & =f_{\text {pert }}^{\text {halo }}(R) \Phi_{0}(R) \cos 2 \theta
\end{aligned}
$$

(see eqs. [1] and [3]), and (repeated from eq. [29])

$$
\Phi_{\text {pert }}^{\text {disk }}(R, \theta)=-f_{\text {pert }}(R) \eta(R) \cos 2 \theta .
$$

The physical interpretation of these equations is that the disk response is proportional to the net perturbation $f_{\text {pert }}$, which is itself the sum of the disk response and the imposed halo perturbation. The disk response is opposite in sign to the halo perturbation, so $f_{\text {pert }}$ in the self-consistent solution must be reduced with respect to the imposed halo perturbation.

The self-consistent solution can be obtained by collection equations (30)-(33) as

$$
f_{\text {pert }}(R)=f_{\text {pert }}^{\text {halo }}(R) \frac{1}{1+\eta(R) / \Phi_{0}(R)} .
$$

In other words, the response of the disk causes the overall potential perturbation to be reduced by a factor of $1+\eta(R) / \Phi_{0}(R)$. All

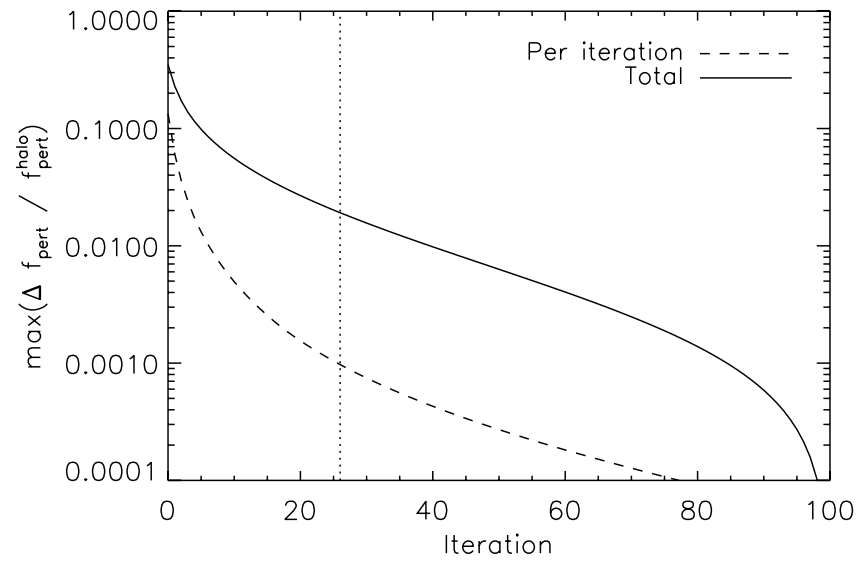

FIG. 1.-Maximum change in the solution for $f_{\text {pert }} / f_{\text {pert }}^{\text {halo }}$ per iteration (dashed line) and maximum difference between the solution at a given iteration and the true solution, assumed to have converged after 100 iterations (solid line), for the fiducial halo and $3 \times 10^{9} M_{\odot}$ disk of $\S 3.1$. The vertical dotted line indicates the point where our convergence criterion is achieved.

terms on the right-hand side have been tabulated at the grid points $R_{i}$, resulting in a trivial evaluation of $f_{\text {pert }}(R)$.

Armed with this new estimate of $f_{\text {pert }}$, we can reexamine equation (28), substitute $f_{\text {pert }}^{\text {proxy }}=f_{\text {pert }}$, and calculate a new value of $\eta(R)$ and therefore of $f_{\text {pert }}(R)$. We repeat this procedure until the maximum change between iterations in the quantity $f_{\text {pert }} / f_{\text {pert }}^{\text {halo }}$, which is a robust indicator of the relative error in $f_{\text {pert }}$, is less than $10^{-3}$ at all radii; this is typically achieved in $20-30$ iterations. We have confirmed for some specific cases that our solution agrees to within $\sim 2 \%$ of the true solution (assumed to have converged after a very large number of iterations) at all radii and to much higher precision at most radii (see Fig. 1). Adopting a stricter convergence criterion has no effect on our results. The relatively large number of iterations is required in order to accurately capture the sharp feature where $f_{\text {pert }} \rightarrow 0$ that is seen in the solutions (see $\S 3$ ).

Given $f_{\text {pert }}$, the disk ellipticity $\epsilon_{\text {disk }}$ can be calculated as a function of radius directly from equation (22), and the forms of the closed orbits can be calculated from equations (11)-(14). This provides a complete description of the disk.

\section{RESULTS}

In this section we demonstrate how the disk dilutes the elliptical potential of the halo and give examples of the net ellipticity induced in the disk. In $\S 3.1$ we demonstrate the main features of the disk-halo systems using a halo with constant axis ratios, while in $\S 3.2$ we investigate how these results are affected by varying disk and halo parameters such as the halo concentration, axis ratio, disk scale length, and run of halo axis ratio with radius.

\subsection{Response for Various Disk Masses}

We demonstrate the main features of our models using a fiducial triaxial NFW96 halo with mass $M_{200}=10^{12} M_{\odot}$, axis ratios $b / a=0.8$ and $c / a=0.6$, and a concentration $c_{200}=12 .{ }^{7}$ These values are typical for galaxy-sized dark matter halos in cosmological simulations (Allgood et al. 2006). The disk rotation axis is aligned with the minor axis of the halo, in agreement with the orientation of the halo angular momentum in simulations (Bailin \& Steinmetz 2005). All disks have radial scale lengths $R_{d}=3.0 \mathrm{kpc}$, with masses that range from zero up to $10^{11} M_{\odot}$.

\footnotetext{
7 The terms $M_{200}$ and $c_{200}$ refer to the mass and concentration relative to the radius $r_{200}$, defined such that the mean density within $r_{200}$ is 200 times the critical density. We assume the Hubble parameter $h=0.7$.
} 


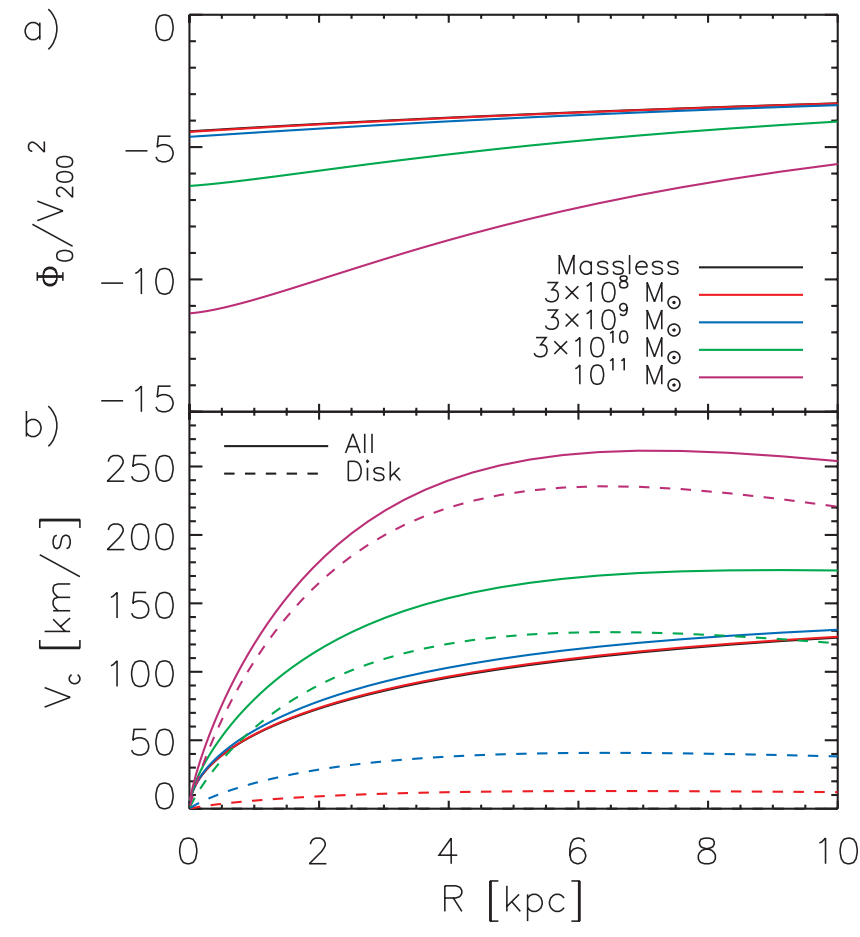

FIG. 2.- (a) Axisymmetric component of the potential, $\Phi_{0}(R)$, for massless disks (black line) and disks of mass $3 \times 10^{8} M_{\odot}$ (red line), $3 \times 10^{9} M_{\odot}$ (blue line), $3 \times 10^{10} M_{\odot}$ (green line), and $10^{11} M_{\odot}$ (purple line) within the fiducial halo of $\S 3.1$. (b) Circular velocity curve within the unperturbed potential. The disk contribution to the rotation curve is denoted with dashed lines. Colors are the same as in $(a)$.

Figure 2 demonstrates how the axisymmetric component of the potential, $\Phi_{0}(R)$, and the rotation curve, $V_{c}(R)$, vary as functions of disk mass. The potential is given in units of $V_{200}^{2} \equiv G M_{200} / r_{200}$. For disk masses less then $3 \times 10^{9} M_{\odot}$, the halo dominates the axisymmetric component of the potential at all radii, with the disk becoming increasingly more important with increasing disk mass beyond this. The nonaxisymmetric component of the potential is demonstrated in Figure 3 for the $3 \times 10^{9} M_{\odot}$ disk. Although the ellipticity of isopotential surfaces, $\epsilon_{\Phi}$, rises to small radii, the magnitude of the potential perturbation, $f_{\text {pert }}^{\text {halo }}$, must vanish at small radii because $\Phi_{x}(R=0)=\Phi_{y}(R=0)$ while $\Phi_{0}(R=0)$ reaches a finite value.

Figure $4 a$ demonstrates both the initial halo perturbation ( $f$ pert , solid lines) and the net perturbation after including the selfconsistent response of the disk ( $f_{\text {pert }}$, dashed lines). Note that even though the halo is identical in each case, $f_{\text {pert }}^{\text {halo }}$ is lower for higher mass disks because the disk contributes to $\Phi_{0}$.

HN06 proposed that a suitable perturbation

$$
f_{\text {pert }}=f_{\text {iso }}(R) \approx a x e^{-x / b}
$$

(with $x \equiv R / r_{s}, a=0.1$, and $b=0.098$ ) would cause the rotation curve of a perturbed NFW96 profile to mimic that of a cored isothermal profile. We denote this as a dotted line in Figure $4 a$. The form of this perturbation is very different from the form of the perturbation that we find to be induced by a triaxial halo of uniform axis ratio, particularly once the self-consistent response of the disk is taken into account.

Figure $4 b$ demonstrates the degree to which the initial halo perturbation $f_{\text {pert }}^{\text {halo }}$ is diluted by the self-consistent response of the disk. This is equal to $f_{\text {pert }} / f_{\text {pert }}^{\text {halo }}$ and is determined from $1 /[1+$ $\left.\eta(R) / \Phi_{0}(R)\right]$. The ellipticity in the potential vanishes in the

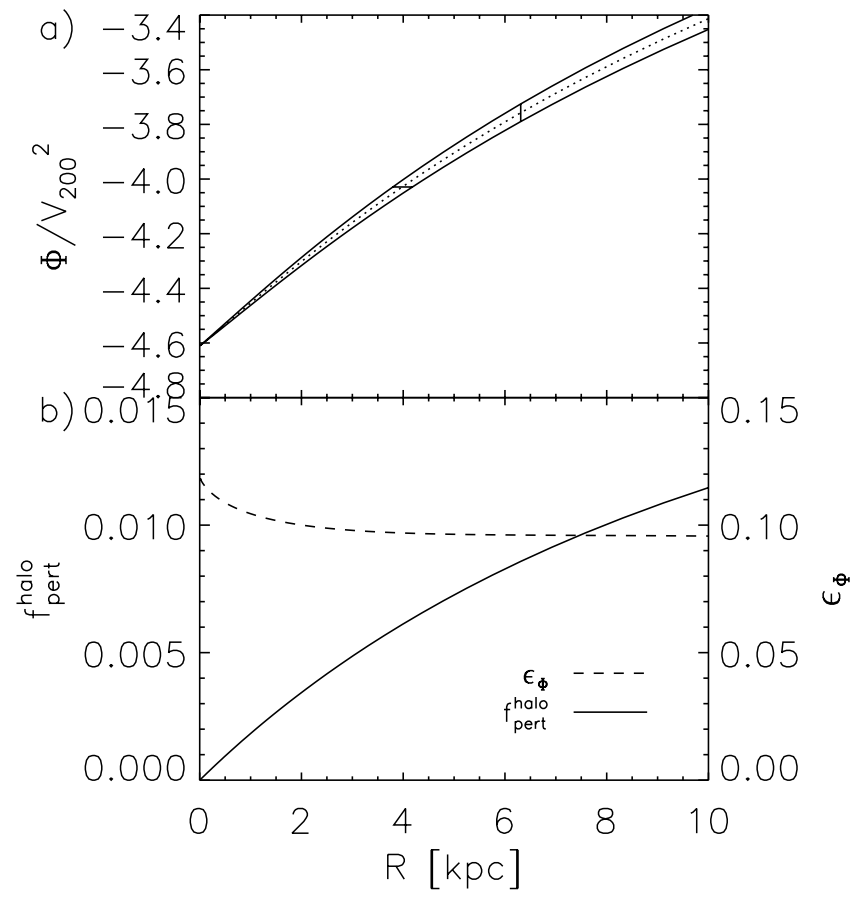

FIG. 3.- (a) Solid lines indicate the potential along the $x$ - (bottom line) and $y$-axis (top line) for the disk of mass $3 \times 10^{9} M_{\odot}$ within the fiducial halo of $\S 3.1$. The dotted line indicates the axisymmetric component of the potential. The horizontal and vertical lines demonstrate how the ellipticity of the isopotential surfaces and the magnitude of the perturbation, respectively, are calculated. (b) Magnitude of the perturbation $f_{\text {pert }}$ (solid line) and ellipticity of the potential $\epsilon_{\Phi}$ (dashed line) for the same halo as in (a). [See the electronic edition of the Journal for a color version of this figure.]

central region where $\eta(R) \gg \Phi_{0}(R)$. Even for a negligible disk mass, the potential in the innermost region is circularized. This region is larger for more realistic disks, which have a significant impact on the potential out to several disk scale lengths. As the disk mass increases, the form of the disk dominates both $\eta(R)$ and $\Phi_{0}(R)$, and therefore, this function approaches an asymptotic form.

Comparison between Figure $4 b$ and the equivalent Figures 2 and 3 of $\mathrm{J} 2 \mathrm{~K}$ reveals dramatically different behavior at small radii: in $\mathrm{J} 2 \mathrm{~K}$, the "reduction factor" reaches a minimum at $1.42 R_{d}$ $\left(=4.26 \mathrm{kpc}\right.$ for $\left.R_{d}=3.0 \mathrm{kpc}\right)$ and then rises to unity, while in Figure $4 b$ it falls monotonically to vanish at small radii. This is a direct result of the radial variation of $f_{\text {pert }}$ in a physically realistic elliptical halo. Because $\eta(R)$ depends inversely on $f_{\text {pert }}$ (see eq. [28]), which must vanish at small radii, $\eta(R)$ must dominate over $\Phi_{0}(R)$ in the inner regions and the potential must become completely circularized.

In Figure $4 c$ we plot the ellipticity of the disk isodensity contours ( $\epsilon_{\text {disk }}$, solid lines $)$ and of the orbits within the disk $\left(\epsilon_{\text {orbit }} \equiv\right.$ $f_{\text {pert }} a_{12}$, dashed lines). For a massless disk, we recover the results of HN06 that the ellipticity rises toward the center of the halo. However, the presence of a massive disk changes the situation dramatically. Because $f_{\text {pert }} / f_{\text {pert }}^{\text {halo }}$ vanishes at small radii (Fig. $4 b$ ), the equilibrium disk is axisymmetric at small radii. Even very low mass disks, which contribute negligibly to $\Phi_{0}$, still respond strongly enough to the elliptical potential to cause an important change in the behavior at small radii. We also note that the ellipticity of disk isophotes are always greater than the ellipticity of orbits within the disk.

\subsection{Varying Disk and Halo Parameters}

HNS07 found that the radial variation of the shape of the potential of cosmological $N$-body halos is not consistent with 


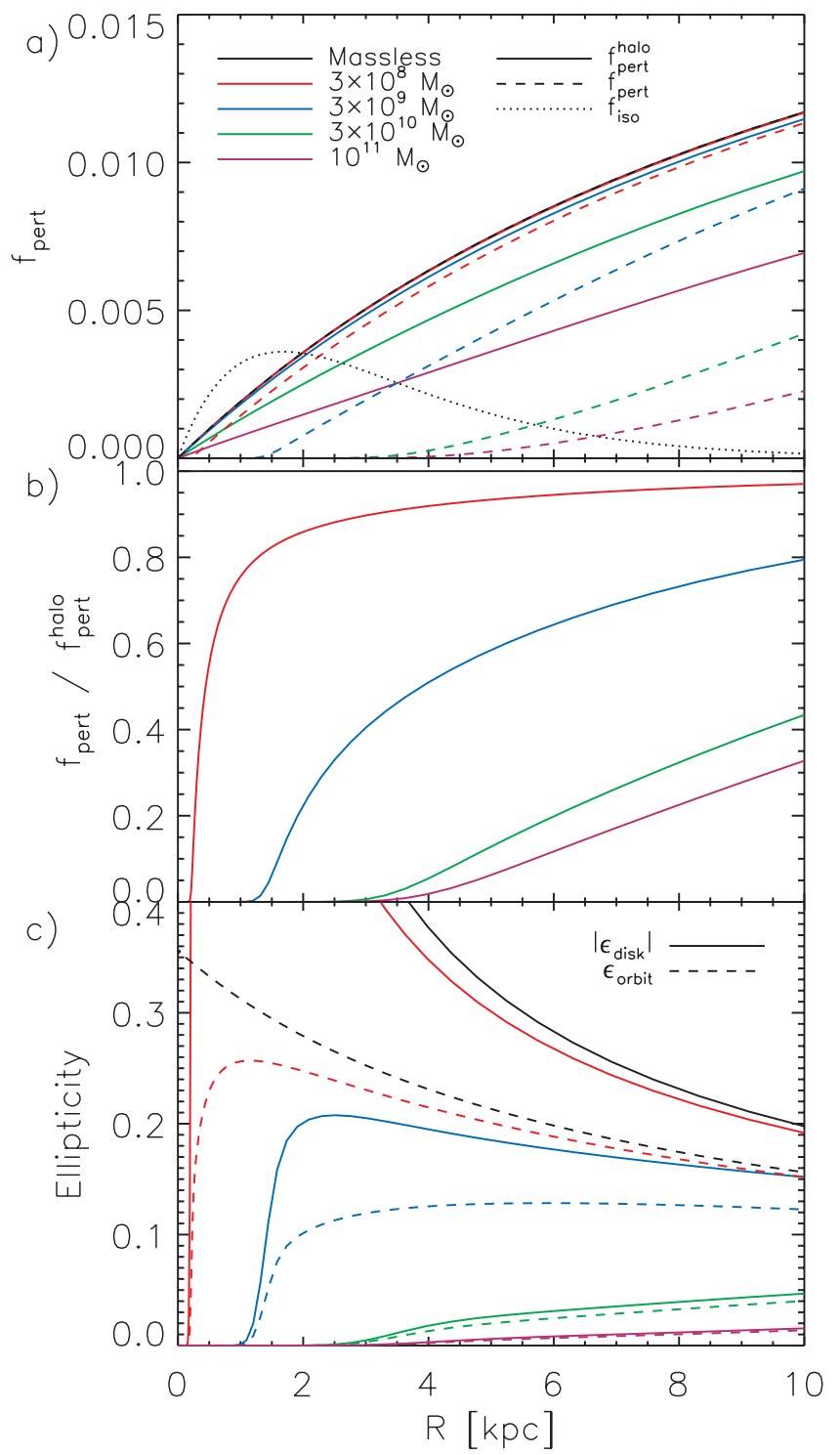

FIG. 4.-(a) Magnitude of the elliptical perturbation in the potential due to the triaxiality of the halo ( $f_{\text {pert }}^{\text {halo }}$, solid lines) and the net perturbation after including the self-consistent response of the disk ( $f_{\text {pert }}$, dashed lines). Different disk masses are indicated by different colors as in Fig. 2. The dotted line shows the form of $f_{\text {pert }}$ proposed by HN06 to produce a rotation curve mimicking a cored isotherma density profile. (b) Ratio by which the input perturbation $f_{\text {pert }}^{\text {hal }}$ becomes diluted due to the self-consistent response of the disk. (c) Ellipticity of the disk isodensity contours $\left(\left|\epsilon_{\text {disk }}\right|\right.$, solid lines; note that $\epsilon_{\text {disk }}$ is negative for positive $f_{\text {pert }}$ in the sign convention we have chosen) and of orbits within the disk ( $\epsilon_{\text {orbit }}$, dashed lines $)$.

self-similar isodensity contours (see also Jing \& Suto 2002; Bailin $\&$ Steinmetz 2005). They found instead that the isopotential axis ratios are well fit by the function

$$
\log \left(\frac{b}{a} \text { or } \frac{c}{a}\right)=\alpha\left[\tanh \left(\gamma \log \frac{r}{r_{\alpha}}\right)-1\right] .
$$

We have recomputed the self-consistent response of disks of varying mass in a potential of this form, with the values of the parameters taken from halo G4 of HNS07, which has a very similar mass and concentration to the halo used in $\S 3.1$. The results are shown in Figure 5. As noted by HNS07, the perturbation in this case contains a peak at intermediate radius and is much more similar to the form of $f_{\text {iso }}$ required by HN06 to fit

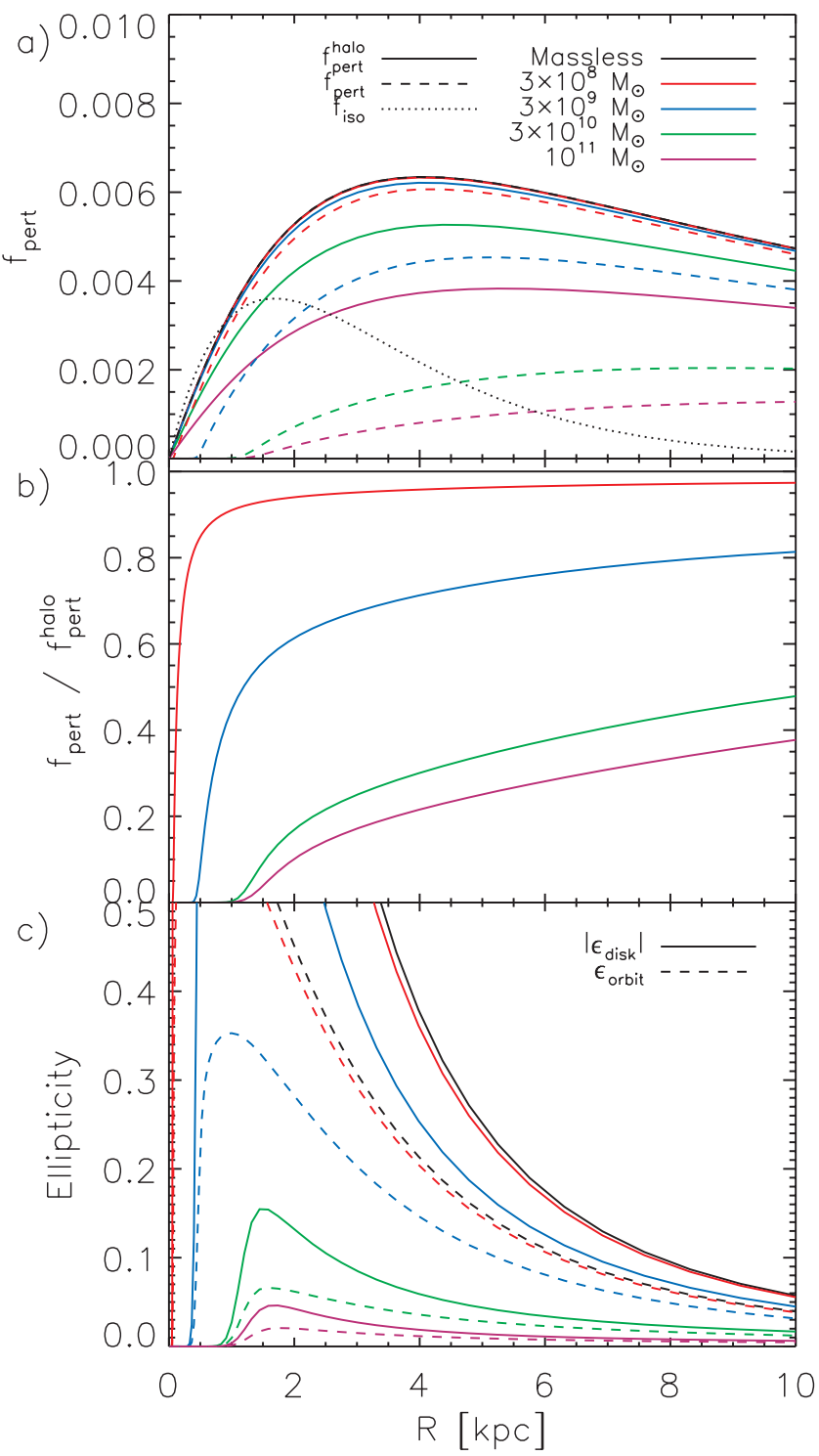

FIG. 5.- Same as Fig. 4, but for disks in a halo potential with the same radial variation of its axis ratios as halo G4 of HNS07.

LSB rotation curves, although unlike $f_{\text {iso }}$ the perturbation remains more prominent to large radius. However, in many cases LSB rotation curves are only measured out to radii of a few $\mathrm{kpc}$, so it is less clear what the required form of $f_{\text {iso }}$ is at larger radii. The disks have less effect on the perturbation than in $\S 3.1$; in particular, the radius inside which they circularize the potential is reduced, resulting in significantly more elliptical orbits at $1-3 \mathrm{kpc}$ than for the equivalent disks in a halo with constant axis ratios. Disk masses higher than $\sim 3 \times 10^{9} M_{\odot}$ reduce the prominence of the peak in the perturbation and shift it to larger radii. The peak in $f_{\text {pert }}^{\text {halo }}$ could be moved to a smaller radius and, therefore, brought further into agreement with $f_{\text {iso }}$, by reducing the $r_{\alpha}$ parameter in equation (36); however, this is unlikely to be a common situation, as G4 already has by far the lowest $r_{\alpha}$ value of any of the halos studied by HNS07.

The effect of the baryonic disk on the shape of the halo is not yet well understood. Simulations suggest that halos containing baryonic disks are less elliptical than halos composed purely of dark matter and that the circularization of the halo occurs most strongly at the center (Kazantzidis et al. 2004). If the intrinsic 


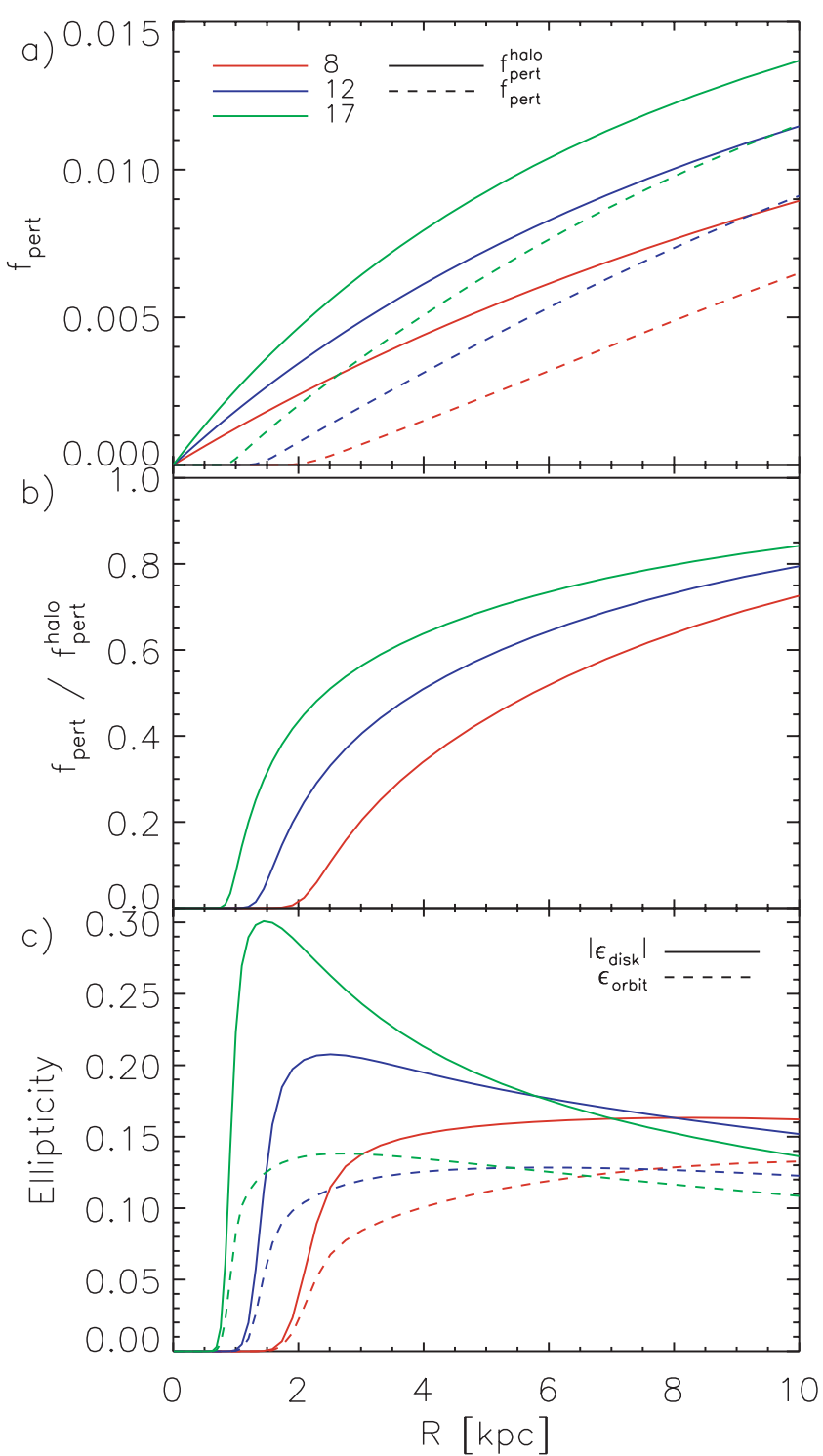

FIG. 6.-(a) Magnitude of the net elliptical perturbation in the potential (dashed lines) and the perturbation due to only the halo (solid lines) for disks of mass $3 \times 10^{9} M_{\odot}$ in halos with concentrations $c_{200}=8$ (red lines), 12 (blue lines), and 17 (green lines). The blue lines in these plots are identical to the blue lines in Fig. 4. (b) Ratio by which the input perturbation becomes diluted due to the response of the disk. (c) Ellipticity of the disk isodensity contours (solid lines) and of orbits within the disk (dashed lines).

shape of the pure dark matter halo is well described by the HNS07 form, which is most elliptical at the center, baryonic processes may result in a situation more similar to the constant axis ratio case of $\S 3.1$. We therefore expect that the regions in which these models differ most strongly, $1-3 \mathrm{kpc}$, are also the regions where the unknown effect of disk formation introduces the most uncertainty into our models.

In order to investigate how other properties of the halo and disk affect our results, we have recalculated the results of $\S 3.1$ (where the halo axis ratio was assumed to be constant with radius) for the $3 \times 10^{9} M_{\odot}$ disk while varying the halo concentration, the $b / a$ axis ratio, the halo mass, and the disk scale length. In more concentrated halos (Fig. 6), the strength of the perturbation due to the halo, $f$ pert , is larger. The disk is also less able to dilute the perturbation in more concentrated halos. The axis ratio of the halo (Fig. 7) has a strong effect on the magnitude of the perturbation, but has virtually no effect on the degree to which

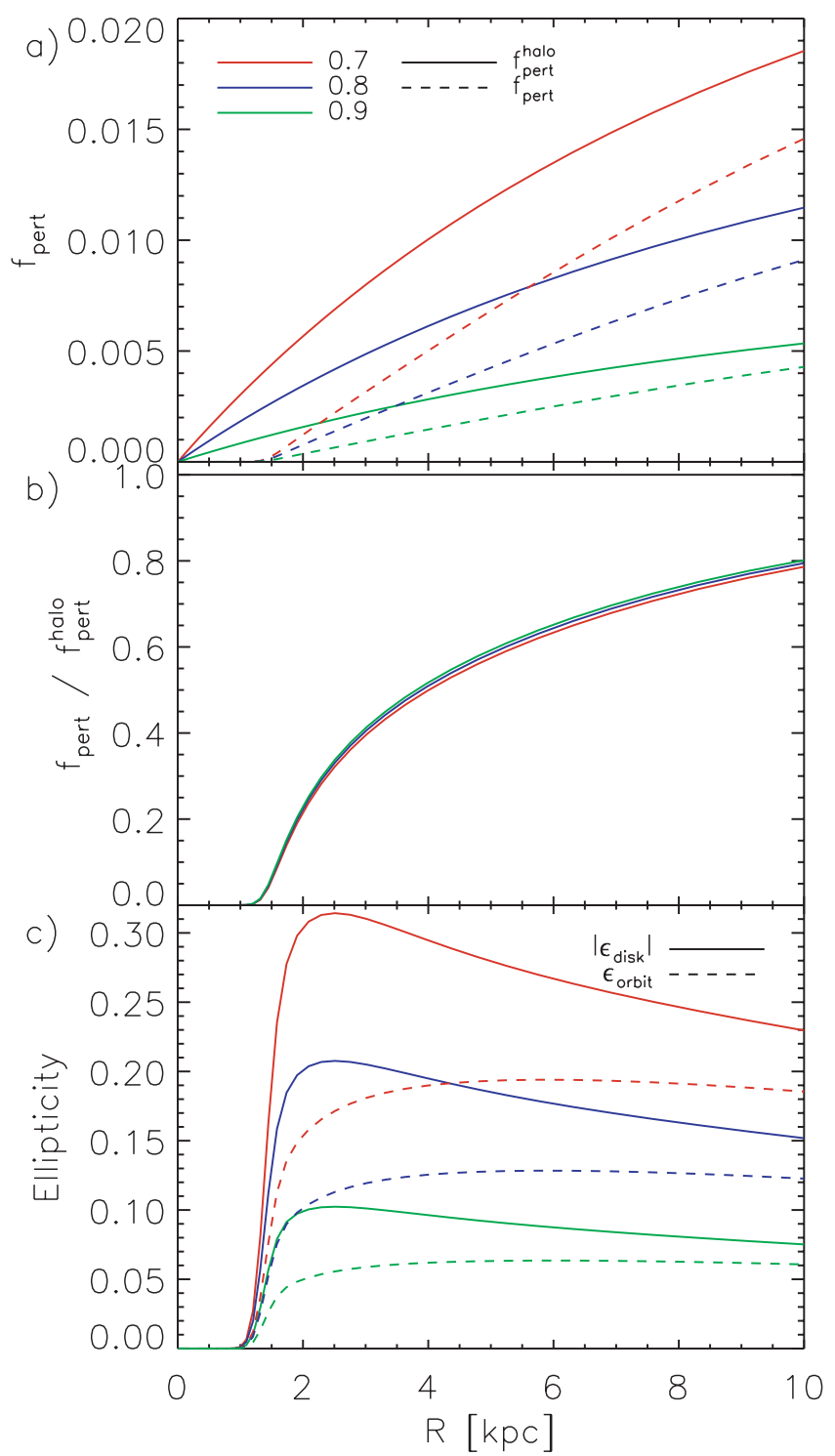

FIG. 7.- Same as Fig. 6, but for halos with axis ratios $b / a=0.7$ (red lines), 0.8 (blue lines), and 0.9 (green lines).

the disk dilutes the perturbation. In Figure 8 we compare halos of different virial mass, $M_{200}$. In order to facilitate comparison between systems of different mass, we have kept $r_{s}$ constant by varying $c_{200}$ in proportion to $r_{200}$ and kept the relative mass of the disk and halo constant. We find that the mass of the halo has little effect on the relative strength of the perturbation (either before or after the disk is taken into account), but the resulting disk ellipticities are higher in lower mass systems. Finally, although the equilibrium shape of the potential is similar regardless of the disk scale length (Fig. 9), a greater ellipticity is required to achieve this reduction in the perturbation for less concentrated disks, i.e., those with larger scale lengths.

\section{DISCUSSION}

\subsection{Impact of Triaxial Halos on the Cusp/Core Problem}

In Figure 10 we plot the azimuthal velocities along the major and minor axes of the halo within the disk plane for disks of different mass in the fiducial sample halo studied in $\S 3$. Full analysis of the velocity fields of these disks will be presented in a 


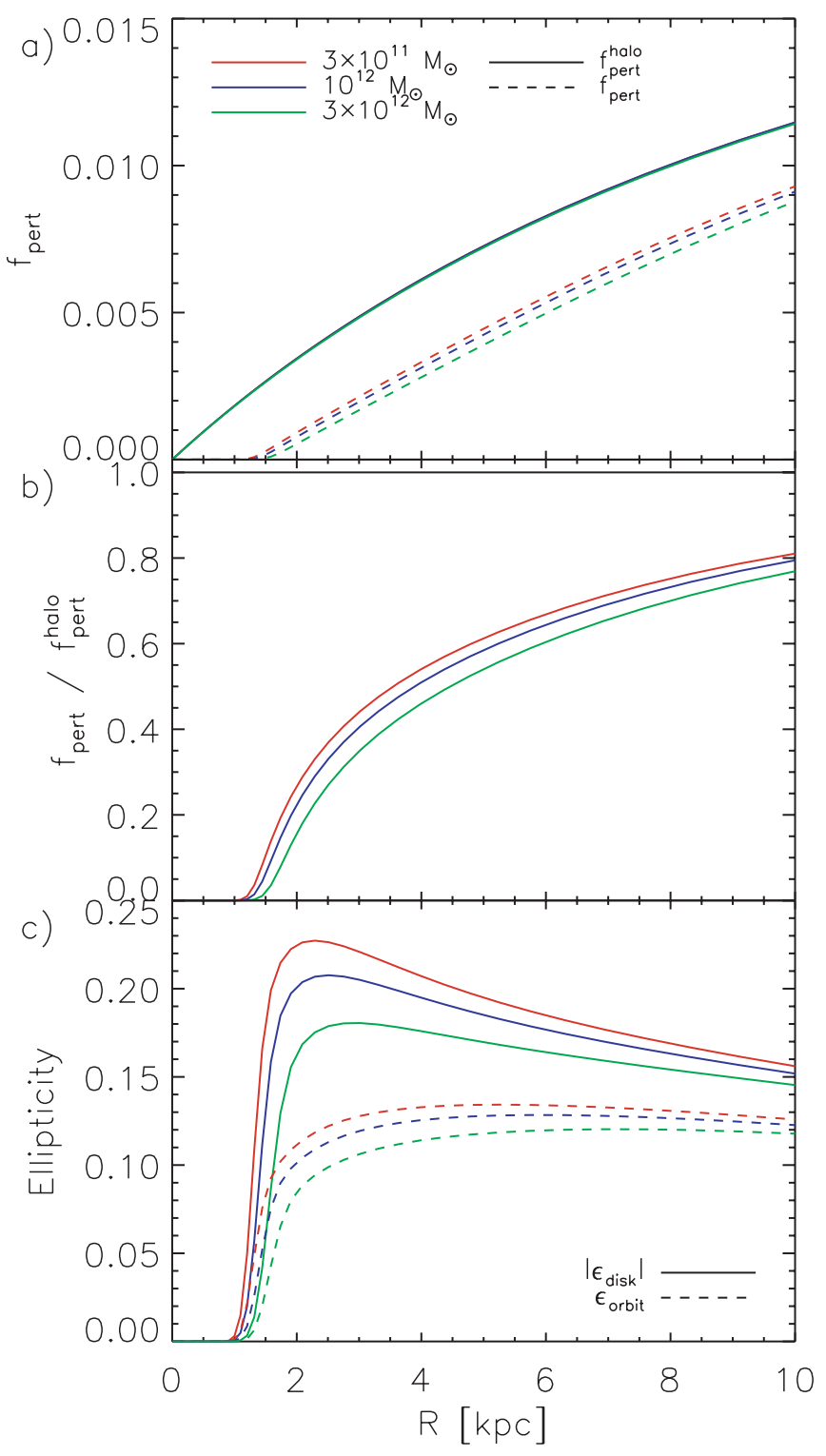

FIG. 8. - Same as Fig. 6, but for halos with virial masses $M_{200}=3 \times 10^{11} M_{\odot}$ (red lines), $10^{12} M_{\odot}$ (blue lines), and $3 \times 10^{12} M_{\odot}$ (green lines). In order to facilitate the comparison, the halo scale radius $r_{s}$ is kept constant by varying $c_{200}$ in proportion to $r_{200}$, and the ratio between the disk and halo mass is kept constant.

future paper. However, we note that none of these rotation curves show the linear rise characteristic of a constant density core, as expected given the dramatic difference between the form of $f_{\text {pert }}$ in these disks and the form of $f_{\text {iso }}$ required by HN06.

If we use the radial variation of the shape of the halo potential proposed by HNS07 (Fig. 11), for which $f_{\text {pert }}^{\text {halo }}$ is much more similar to $f_{\text {iso }}$, we find that for very low disk masses the azimuthal velocity along the minor axis of the halo is characterized by a much more gradual rise. Observationally, such a rotation curve might be interpreted as indicating a constant-density core in the dark matter halo. However, for disk masses above $3 \times 10^{9} M_{\odot}$ $(0.3 \%$ of the virial mass of the halo and just $1.8 \%$ of the baryonic mass of the system) the response of the disk removes this feature from the center of the rotation curve. Based on these results, we conclude that simple analyses of the shapes of halos are therefore not sufficient to determine whether halo triaxiality can reconcile LSB rotation curves with cuspy halo density profiles, as suggested by HN06; full analyses that take into account the disk response are

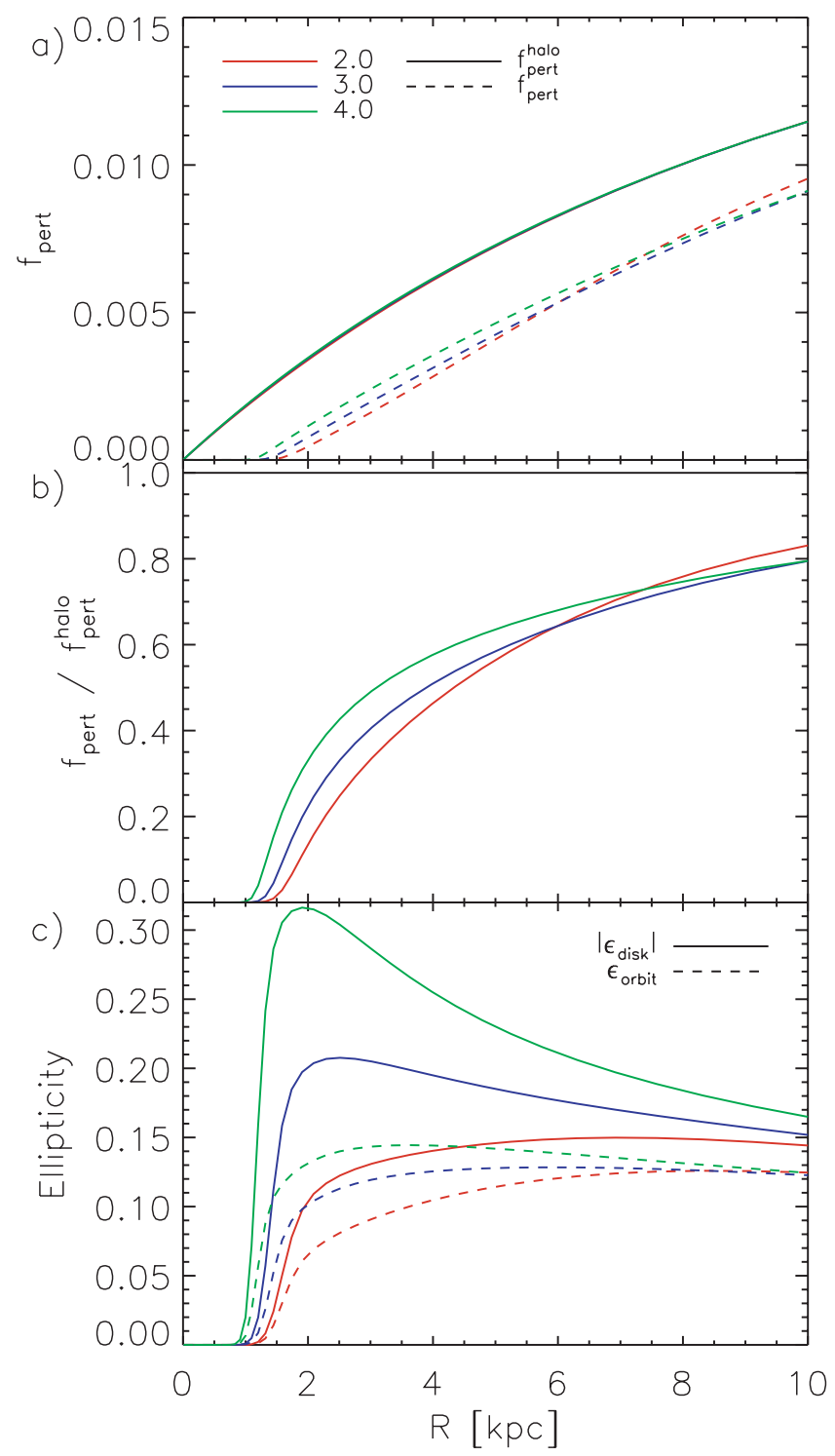

FIG. 9.- Same as Fig. 6, but for disks with scale lengths $R_{d}=2.0$ (red lines), 3.0 (blue lines), and 4.0 (green lines).

required. Preliminary tests on simulated velocity fields constructed using the results of this paper do suggest that triaxiality can produce apparent constant-density cores, in agreement with HN06, but a more detailed analysis including many halos and lines of sight is needed before comparing to the observational distribution of density profile slopes (Simon et al. 2005).

We also plot the maximum radial velocity (amplitude of noncircular motions) at each radius in Figures 10 and 11. Although the radial velocities are much smaller than the azimuthal velocities in all but the lowest mass disks, they are at a level that can be detected in observations of two-dimensional velocity fields. The magnitude of the radial motions, which reach $5-35 \mathrm{~km} \mathrm{~s}^{-1} \mathrm{de}-$ pending on the disk mass and halo properties, are consistent with the magnitude of radial motions found by Simon et al. (2005) and reproduce the observed trend for the radial motions to be negligible at small radii and to only become important at larger radii.

\subsection{Comparison to Observed Disk Ellipticities}

It is interesting to compare the ellipticity of our model disks to observed values. Using two-dimensional velocity fields, Simon 


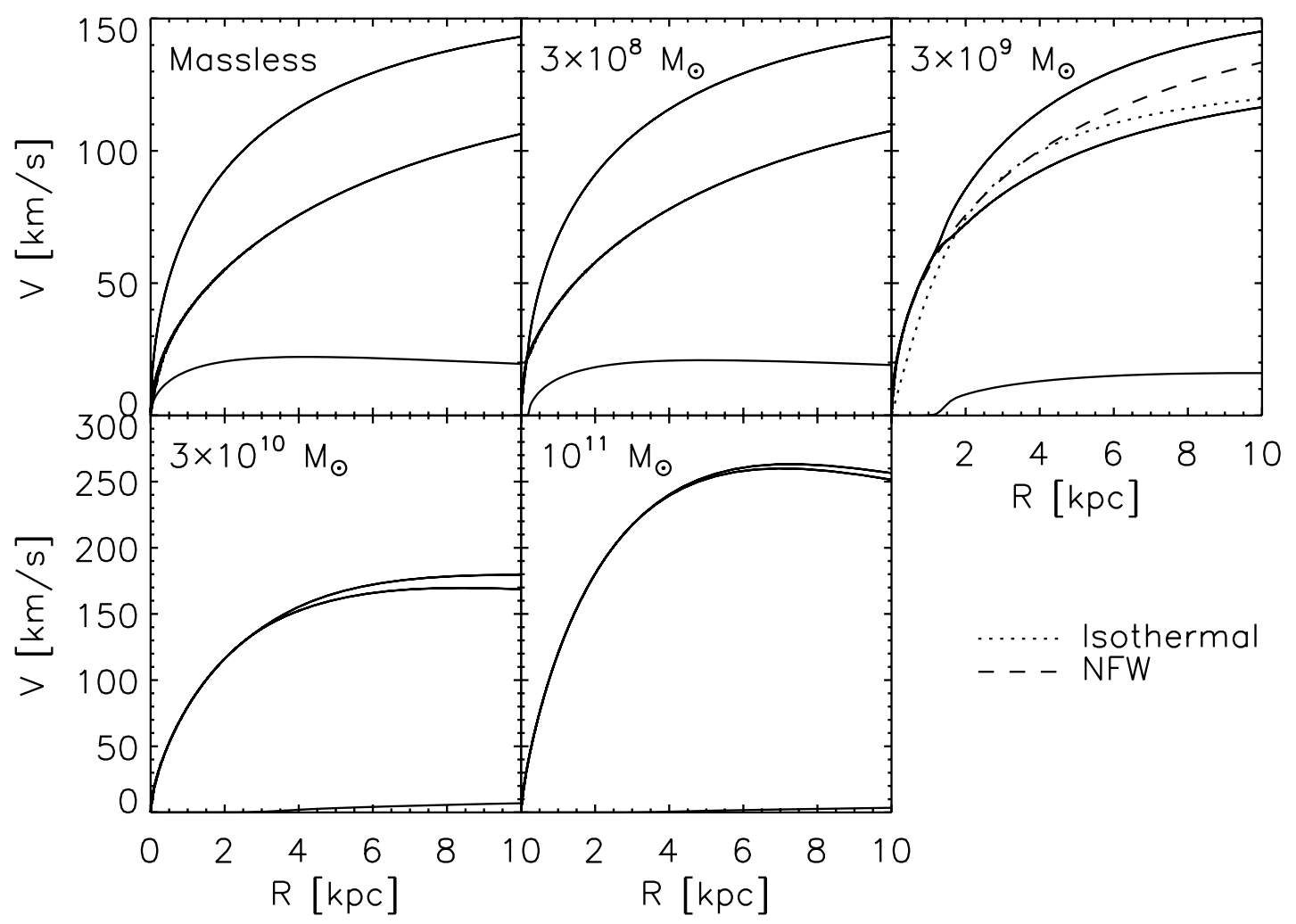

FIG. 10.- Azimuthal velocity along the halo major axis (top line) and minor axis (middle line) and the maximum radial velocity at each radius (bottom line) for disks of varying mass in the halo of $\S 3.1$. NFW96 and isothermal rotation curves are shown for reference in the top right panel. [See the electronic edition of the Journal for a color version of this figure.]

et al. (2005) found lower limits on the orbital ellipticities ranging from 0 up to 0.175 , similar to the orbital ellipticities in our model disks. We note that the orbital ellipticity throughout most of the disk is determined by the mass of the disk (Fig. 4c), the ellipticity of the halo (Fig. 7c), and the variation of halo ellipticity with radius (compare Figs. $4 c$ and $5 c$ ), with very little dependence on the concentration of either the halo (Fig. 6c) or the disk (Fig. 9c) or on the global mass of the system (Fig. 8c). We therefore predict that galaxies with large observed ellipticities such as NGC 4605 either lie in halos that are more triaxial than average near their center or contain an unusually low fraction of their mass in their disk. The trends in intrinsic disk ellipticity that we predict may be tested with further analysis of larger kinematic samples, such as those presented by Ganda et al. (2006).

Ryden (2006) found that the distribution of isophotal shapes of galaxies in the 2MASS Large Galaxy Atlas (Jarrett et al. 2003), as measured in the near-infrared $K_{s}$ band (which is a good tracer of the stellar disk mass), is well fit if the intrinsic disk ellipticity distribution is a truncated Gaussian distribution centered at 0.01 with a width of $\sigma=0.26$. This corresponds to a median ellipticity of 0.18 , with $68 \%$ of disks having ellipticities $0.05 \leq \epsilon_{\text {disk }} \leq 0.37$. For the disk parameters we have studied, the $18.8 \mathrm{mag} \mathrm{arcsec}^{-2}$ isophote at which her shapes were measured corresponds to radii of between 1.5 and $8.5 \mathrm{kpc}$. Our models naturally produce disk ellipticities in this range at these radii, although the highest ellipticities can only be produced by our least massive disks in our most elliptical potentials.

Finally, we note that our models only take into account ellipticity in the disk induced by the dark matter halo. Central regions of the disk, which have high surface density and sit in an axisymmetric potential, may be unstable to bar formation (Berentzen \& Shlosman 2006). This can induce additional el- lipticity to the kinematic and photometric properties of disk galaxies (e.g., Valenzuela et al. 2007).

\section{CONCLUSIONS}

We have presented a computationally efficient method to selfconsistently determine the dynamics of massive disks in triaxial dark matter halos. Our work extends the study of J2K by allowing the perturbation to the potential to vary with radius in an appropriate manner and by allowing the ellipticity of the disk to vary with radius self-consistently. These improvements result in qualitatively different behavior for the ellipticity of disks at small radii: J2K found that disks counteract the halo ellipticity most strongly at $1.42 R_{d}$ and have a negligible effect at small radii; in contrast, we find that the effect of the disk increases monotonically to small radii, completely circularizing the potential in the innermost regions.

This self-consistent radially varying response of the disk to the halo perturbation must be taken into account when comparing the observed kinematic and photometric properties of galactic disks to those expected in triaxial dark matter halos, particularly for comparisons at small radii. When this response is calculated for plausible halo values, model disks have ellipticities consistent with those determined from observations of velocity fields and from isophotal axis ratio distributions. We also find that the radial variation of the halo axis ratios has a significant impact on the disk structure. Halos with axis ratios that vary with radius as suggested by cosmological simulations produce much more elliptical orbits in the inner disk than do halos with constant axis ratios, resulting in potential perturbations similar to the perturbation required to create apparent cores in galaxy density profiles. Further analysis exploring in detail the conditions under which corelike rotation 


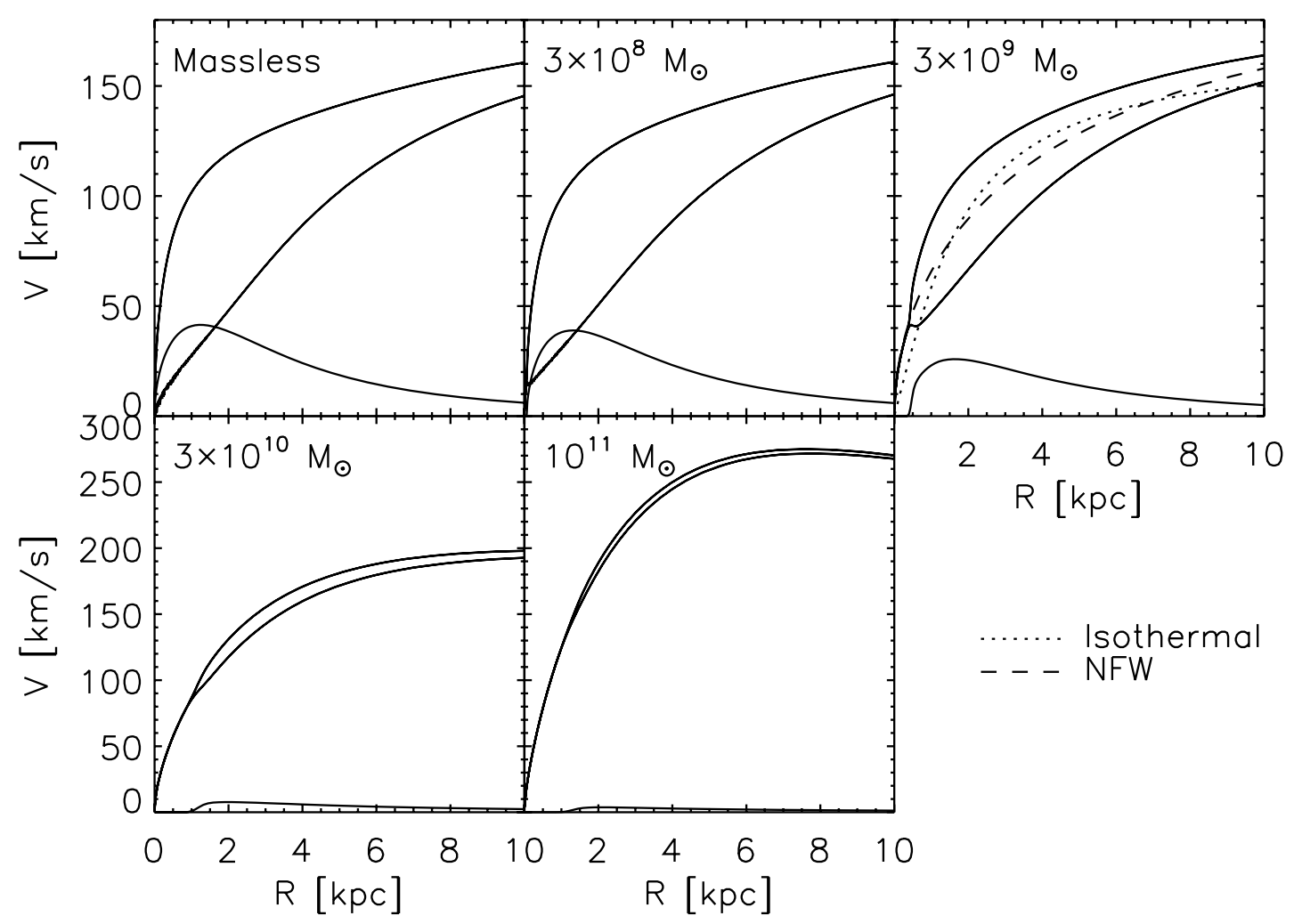

FIG. 11.-Same as Fig. 10, but for the shape of the halo potential found by HNS07 for their halo G4. [See the electronic edition of the Journal for a color version of this figure.]

curves might be obtained will be necessary to determine if halo triaxiality can resolve the cusp/core problem.

J. B. thanks the Australian Research Council for financial support. J. D. S. acknowledges the support of a Millikan Fellow- ship provided by Caltech. B. K. G. and C. P. gratefully acknowledge the support of the Australian Research Councilsupported "Commonwealth Cosmology Initiative," DP 0665574. We thank Miloš Milosavljević and Darren Croton for helpful conversations and the referee, Chanda Jog, for a very useful report.

\section{APPENDIX A}

\section{SECOND-ORDER TERMS AND $m=4$ DISTORTIONS}

We have assumed that the halo perturbation and the induced distortion in the disk are completely described by the $m=2$ mode. This is a direct consequence of only including terms linear in the small quantities $f_{\text {pert }}, \epsilon_{\text {disk }}$, and their derivatives.

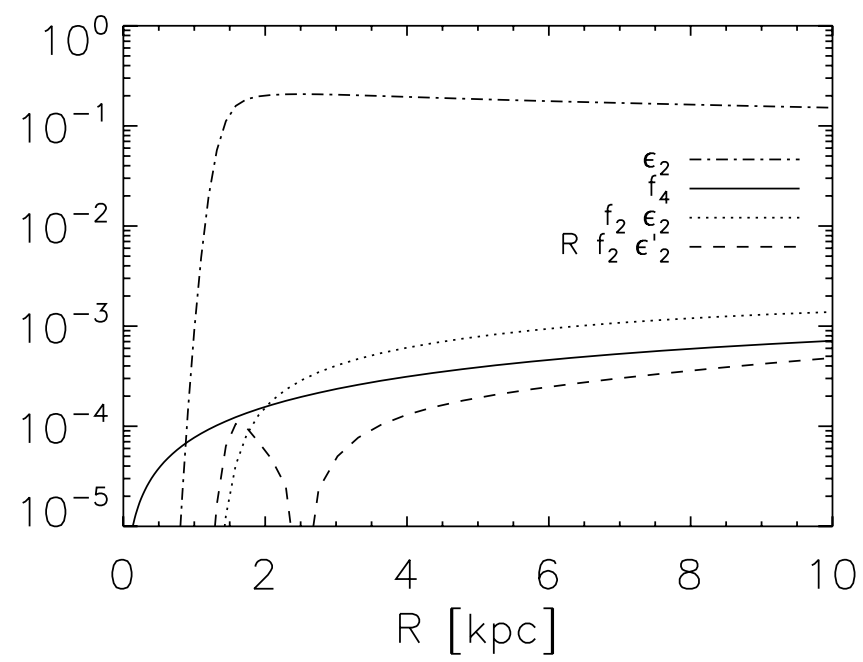

FIG. 12.-Magnitude of terms contributing to the $m=4$ distortion in the disk compared to $\epsilon_{2}$, the magnitude of the calculated $m=2$ distortion for a disk of mass $3 \times 10^{9} M_{\odot}$ in the fiducial halo of $\S 3.1$. 
The validity of this assumption can be tested by evaluating the magnitude of the second-order terms that contribute to the $m=4$ distortion in the disk. If we expand the potential as

$$
\Phi(R, \theta)=\Phi_{0}(R)\left[1+f_{2}(R) \cos 2 \theta+f_{4}(R) \cos 4 \theta\right],
$$

the disk surface density as

$$
\Sigma(R, \theta)=\Sigma_{0} \exp \left\{-\frac{R}{R_{d}}\left[1-\frac{\epsilon_{2}(R)}{2} \cos 2 \theta-\frac{\epsilon_{4}(R)}{2} \cos 4 \theta\right]\right\}
$$

and include all second-order terms, then the $m=4$ distortion in the disk, $\epsilon_{4}$, depends on terms of order $f_{4}, f_{2} \epsilon_{2}$, and $R f_{2} d \epsilon_{2} / d R{ }^{8}$

Figure 12 compares the magnitude of these terms to the $m=2$ ellipticity for the $3 \times 10^{9} M_{\odot}$ disk in the fiducial halo of $\S 3.1$. The higher order terms are more than 2 orders of magnitude smaller than the first-order terms over most of the disk and are also negligible in the central region where the first-order terms vanish, validating our use of linear perturbation theory.

${ }^{8}$ As in the case of the $m=2$ mode, there is also a negligible term of order $R d\left(f_{4} a_{14}\right) / d R$.

\section{REFERENCES}

Allgood, B., Flores, R. A., Primack, J. R., Kravtsov, A. V., Wechsler, R. H., Faltenbacher, A., \& Bullock, J. S. 2006, MNRAS, 367, 1781

Bailin, J., \& Steinmetz, M. 2005, ApJ, 627, 647

Bailin, J., et al. 2005, ApJ, 627, L17

Berentzen, I., \& Shlosman, I. 2006, ApJ, 648, 807

Binney, J., \& Tremaine, S. 1987, Galactic Dynamics (Princeton: Princeton Univ. Press)

de Blok, W. J. G., McGaugh, S. S., Bosma, A., \& Rubin, V. C. 2001, ApJ, 552, L23

Dubinski, J. 1994, ApJ, 431, 617

El-Zant, A. A. 2001, Ap\&SS, 276, 1023

Freeman, K. C. 1970, ApJ, 160, 811

Ganda, K., Falcón-Barroso, J., Peletier, R. F., Cappellari, M., Emsellem, E., McDermid, R. M., de Zeeuw, P. T., \& Carollo, C. M. 2006, MNRAS, 367, 46 Gerhard, O. E., \& Vietri, M. 1986, MNRAS, 223, 377

Hayashi, E., \& Navarro, J. F. 2006, MNRAS, 373, 1117 (HN06)

Hayashi, E., Navarro, J. F., \& Springel, V. 2007, MNRAS, 377, 50 (HNS07)

Jarrett, T. H., Chester, T., Cutri, R., Schneider, S. E., \& Huchra, J. P. 2003, AJ, 125,525
Jing, Y. P., \& Suto, Y. 2002, ApJ, 574, 538

Jog, C. J. 1997, ApJ, 488, 642 1999, ApJ, 522, 661 2000, ApJ, 542, 216 (J2K)

Kazantzidis, S., Kravtsov, A. V., Zentner, A. R., Allgood, B., Nagai, D., \& Moore, B. 2004, ApJ, 611, L73

Navarro, J. F., Frenk, C. S., \& White, S. D. M. 1996, ApJ, 462, 563 (NFW96)

Rix, H.-W., \& Zaritsky, D. 1995, ApJ, 447, 82

Ryden, B. S. 2006, ApJ, 641, 773

Schoenmakers, R. H. M., Franx, M., \& de Zeeuw, P. T. 1997, MNRAS, 292, 349

Simon, J. D., Bolatto, A. D., Leroy, A., Blitz, L., \& Gates, E. L. 2005, ApJ, 621,757

Valenzuela, O., Rhee, G., Klypin, A., Governato, F., Stinson, G., Quinn, T., \& Wadsley, J. 2007, ApJ, 657, 773

Warren, M. S., Quinn, P. J., Salmon, J. K., \& Zurek, W. H. 1992, ApJ, 399, 405 